\title{
Comprehensive force field for multiply-chlorinated hydrocarbons
}

\author{
Sung Hyo Chough* and Samuel Krimm \\ Department of Physics and Macromolecular Research Center, University of Michigan, Ann Arbor, \\ MI 48109, U.S.A.
}

(Received 26 December 1989; in final form and accepted 12 April 1990)

\begin{abstract}
A force field is presented that has been optimized for chlorinated hyrocarbons containing isolated, vicinal, and geminal secondary chlorines. For carbon-chlorine stretch modes, 74 frequencies in 10 molecules are reproduced with an average error of $4.5 \mathrm{~cm}^{-1}$. This force field can therefore serve to study these conformation-dependent modes in multiply-chlorinated hydrocarbons.
\end{abstract}

\section{INTRODUCTION}

IN the previous two papers $[1,2]$ we presented the results of force field refinements for vicinal and geminal secondary chlorides, as derived from vibrational analyses of racemic and meso 2,3,4-trichloropentanes and trans, gauche and gauche' 2,2,3-trichlorobutanes, respectively. In the former case [1], the refinement was optimized to include data on 2chlorobutane, 3-chloropentane, and racemic and meso 2,4-dichloropentanes [3-5], as well as the vicinal chlorines of racemic and meso 2,3-dichlorobutane [6]. In the latter case [2], data on 2,2-dichloropropane [7] and trans and gauche 2,2-dichlorobutane [8] were included in the refinement.

In this paper we present the comprehensive force field encompassed by the above kinds of secondary chloride structures. The general approach to refining this force field was as follows. We developed a program to list all possible environments of each force constant in a chlorinated hydrocarbon chain, focussing on the segments that are likely to occur in chlorinated poly(vinyl chloride). The model compounds listed above were used to refine force constants for many of these different environments, with all molecules containing a given environment being refined together. Since some possible environments were not available in our model compounds, we have (for completeness) tentatively suggested values for these equal to the most closely similar environments for which we have refined constants.

This force field should serve not only to interpret the spectra of multiply-chlorinated hydrocarbonss, but should also be suitable for the analysis of the spectra of chlorinated polymers, such as chlorinated polyethylene, poly(vinyl chloride) [9], and chlorinated poly(vinyl chloride). Since the $\mathrm{CCl}$ stretch frequencies are very sensitive to the local backbone conformation $[10,11]$, they can be an important method of studying chain conformation in such polymers. We also present the observed and calculated frequencies for these modes in the molecules studied. The results for other modes are given in [12].

\section{FORCE FIELD}

The local symmetry coordinates used in the normal mode calculations are defined in Table 1, based on the atom designations of Fig. 1. The structural parameters were: $r(\mathrm{C}-\mathrm{H})=1.09 \AA, r(\mathrm{C}-\mathrm{C})=1.54 \AA$ and $r(\mathrm{C}-\mathrm{Cl})=1.79 \AA$; all angles were assumed to be tetrahedral, and dihedral angles were taken to be $180^{\circ}$ and $\pm 60^{\circ}$ for trans and gauche conformations, respectively. The force field is presented in Table 2.

At first we tried a minimally differentiated force field, i.e. using one force constant, independent of the environment, where possible. For example, a single value was first

\footnotetext{
* Present address: Department of Chemical Engineering, Chonnam National University, Kwangju, Korea 500.
} 
Table 1. Local symmetry coordinates of $\mathrm{CH}_{3}-,-\mathrm{CHX}-$ and $-\mathrm{CX}_{2}-$ in chlorinated hydrocarbons

\begin{tabular}{|c|c|c|c|}
\hline Group & Coordinate & Symbol* & Definition $\dagger$ \\
\hline \multirow[t]{8}{*}{$\mathrm{CH}_{3}$} & Symmetric stretch & M ss & $\mathrm{CH}^{1}+\mathrm{CH}^{2}+\mathrm{CH}^{3}$ \\
\hline & Antisymmetric stretch 1 & $\mathrm{M}$ as 1 & $2 \mathrm{CH}^{1}-\mathrm{CH}^{2}-\mathrm{CH}^{3}$ \\
\hline & Antisymmetric stretch 2 & $\mathbf{M}$ as2 & $\mathrm{CH}^{2}-\mathrm{CH}^{3}$ \\
\hline & Symmetric bend & $\mathrm{M} \mathbf{s b}$ & $\begin{array}{l}\mathrm{H}^{2} \mathrm{CH}^{3}+\mathrm{H}^{1} \mathrm{CH}^{3}+\mathrm{H}^{1} \mathrm{CH}^{2}-\mathrm{CCH} \\
-\mathrm{CCH}^{2}-\mathrm{CCH}^{3}\end{array}$ \\
\hline & Antisymmetric bend 1 & $\mathrm{M}$ ab1 & $2 \mathrm{H}^{2} \mathrm{CH}^{3}-\mathrm{H}^{1} \mathrm{CH}^{3}-\mathrm{H}^{\mathrm{l}} \mathrm{CH}^{2}$ \\
\hline & Antisymmetric bend 2 & Mab2 & $\mathrm{H}^{1} \mathrm{CH}^{3}-\mathrm{H}^{1} \mathrm{CH}^{2}$ \\
\hline & Rock 1 & Mrl & $2 \mathrm{CCH}^{1}-\mathrm{CCH}^{2}-\mathrm{CCH}^{3}$ \\
\hline & Rock 2 & $\mathrm{Mr} 2$ & $\mathrm{CCH}^{2}-\mathrm{CCH}^{3}$ \\
\hline \multirow[t]{7}{*}{$\mathrm{CHX}$} & $\mathrm{CH}$ stretch & $\mathrm{CHs}$ & $\mathrm{CH}$ \\
\hline & CX stretch & $\mathrm{CXs}$ & $\mathrm{CX}$ \\
\hline & $\mathrm{CH}$ out-of-plane bend & $\mathrm{CH}$ ob & $\mathrm{C}^{\prime} \mathrm{CH}-\mathrm{HCC}^{\prime \prime}$ \\
\hline & $\mathrm{CH}$ in-plane bend & $\mathrm{CH}$ ib & $2 \mathrm{HCX}-\mathrm{C}^{\prime} \mathrm{CH}-\mathrm{HCC}^{\prime \prime}$ \\
\hline & CX out-of-plane bend & $\mathrm{CX}$ ob & $\mathrm{C}^{\prime} \mathrm{CX}-\mathrm{XCC}$ \\
\hline & $\mathrm{CX}$ in-plane bend & $\mathrm{CX}$ ib & $\begin{array}{l}\mathrm{C}^{\prime} \mathrm{CX}+\mathrm{XCC}+\mathrm{C}^{\prime} \mathrm{CC}^{\prime \prime}-\mathrm{C}^{\prime} \mathrm{CH} \\
-\mathrm{HCC}-\mathrm{HCX}\end{array}$ \\
\hline & Deformation & CHX def & $\mathrm{C}^{\prime} \mathrm{CC}^{\prime \prime}-\mathrm{C}^{\prime} \mathrm{CX}-\mathrm{XCC}^{\prime \prime}$ \\
\hline \multirow[t]{7}{*}{$\mathrm{CX}_{2}$} & Symmetric stretch & $\mathrm{CX}_{2}$ ss & $\mathrm{CX}+\mathrm{CX}^{\prime}$ \\
\hline & Antisymmetric stretch & $\mathrm{CX}_{2}$ as & $\mathrm{CX}-\mathrm{CX}^{\prime}$ \\
\hline & Deformation & $\mathrm{CX}_{2}$ def & $\begin{array}{l}5 \mathrm{C}^{\prime} \mathrm{CC}^{\prime \prime}-\mathrm{C}^{\prime} \mathrm{CX}-\mathrm{CX}^{\prime} \mathrm{CX}^{\prime}-\mathrm{XCC}^{\prime \prime} \\
-\mathrm{X}^{\prime} \mathrm{CC}^{\prime \prime}-\mathrm{XCX}^{\prime}\end{array}$ \\
\hline & Bend & $\mathrm{CX}_{2} \mathrm{~b}$ & $\begin{array}{l}4 \mathrm{XCX}^{\prime}-\mathrm{C}^{\prime} \mathrm{CX}-\mathrm{C}^{\prime} \mathrm{CX}^{\prime}-\mathrm{XCC}^{\prime \prime} \\
-\mathrm{X}^{\prime} \mathrm{CC}^{\prime \prime}\end{array}$ \\
\hline & Wag & $\mathrm{CX}_{2} \mathrm{w}$ & $\mathrm{C}^{\prime} \mathrm{CX}+\mathrm{C}^{\prime} \mathrm{CX}^{\prime}-\mathrm{XCC}^{\prime \prime}-\mathrm{X}^{\prime} \mathrm{CC}^{\prime \prime}$ \\
\hline & Rock & $\mathrm{CX}_{2} \mathrm{r}$ & $\mathrm{C}^{\prime} \mathrm{CX}-\mathrm{C}^{\prime} \mathrm{CX}^{\prime}+\mathrm{XCC}^{\prime \prime}-\mathrm{X}^{\prime} \mathrm{CC}^{\prime \prime}$ \\
\hline & Twist & $\mathrm{CX}_{2} \mathrm{tw}$ & $\mathrm{C}^{\prime} \mathrm{CX}-\mathrm{C}^{\prime} \mathrm{CX}^{\prime}-\mathrm{XCC}^{\prime \prime}+\mathrm{X}^{\prime} \mathrm{CC}^{\prime \prime}$ \\
\hline
\end{tabular}

${ }^{*} \mathrm{M}=$ methyl, $\mathrm{X}=$ chlorine.

$\dagger \mathrm{CH}^{1}$ is the bond trans to the non-methyl $\mathrm{C}-\mathrm{C}$ bond. $\mathrm{CH}_{2}$ has the same definition as $\mathrm{CX}_{2}$.

assigned to the four $\mathrm{CC}$ stretch constants (see Table 2). In such a case, however, the frequency agreement was poor and we had to allow for different values depending on the environment. Our force field includes all interaction constants between two bonds that have an atom in common and between two angles that have a bond in common, but does not include most interactions between two angles that have only an apex atom in common or that have neither a bond nor an apex atom in common. However, interactions such as those between $\mathrm{CCl}$ stretch and $\mathrm{C}^{\prime} \mathrm{CC}^{\prime \prime}$ bend, between $\mathrm{C}^{\prime} \mathrm{C}$ stretch and $\mathrm{HCC}^{\prime \prime}$ bend, and between $\mathrm{C}^{\prime} \mathrm{CCl}$ bend and $\mathrm{C}^{\prime \prime} \mathrm{CCl}^{\prime}$ bend are included. In cases where three successive $\mathrm{C}$ atoms along the chain have attached $\mathrm{Cl}$ atoms (one or two), some force constants related to the center $\mathrm{C}$ atom were allowed to refine independently; these are specifically indicated in Table 2 . Similar force constants in different environments, such as $\mathrm{CH}_{3}(=\mathrm{M}), \mathrm{CH}_{2}(=\mathrm{HH}), \mathrm{CHCl}(=\mathrm{HX})$ and $\mathrm{CCl}_{2}(=\mathrm{XX})$, were also allowed to refine independently. When specific environments were not available from our model compounds, force constants were assigned from a similar environment so that more complex structures could still be computed. Thus, HXHXXX is among the 11 possible environments for the $\mathrm{CCC}$ bend constant, but it is not represented in our model compounds. We are suggesting in this case that the value for the HXHXHX environment be used.
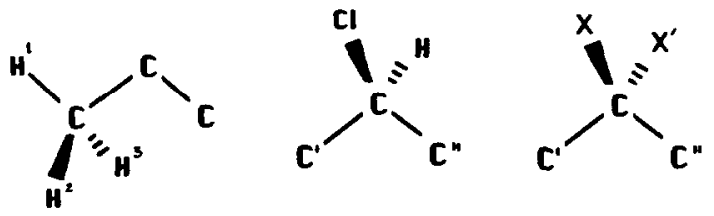

Fig. 1. Designation of atoms in local symmetry coordinates for $-\mathrm{CH}_{3},-\mathrm{CHCl}$ - and $-\mathrm{CCl}_{2}-$ units. 
Table 2. Force constants for chlorinated hydrocarbons

\begin{tabular}{|c|c|c|c|c|c|}
\hline Constant* & Environ. $\dagger^{\dagger}$ & Value & Constant* & Environ. $\dagger$ & Value \\
\hline MH & $\mathrm{ME}$ & 4.7522 & & МЕНХHН & 0.2738 \\
\hline \multirow[t]{2}{*}{$\mathrm{CH}$} & HX & 4.6932 & & MEXXHX & 0.5867 \\
\hline & HH & 4.4870 & & MEXXHH & 0.5867 \\
\hline \multirow[t]{3}{*}{ MC } & MEHH & 4.4858 & & MEHHHX & 0.2691 \\
\hline & MEHX & 4.7047 & & MEHHXX & 0.2691 \\
\hline & MEXX & 4.2576 & $\mathrm{CC}, \mathrm{CC}$ & HXHXHX & 0.2248 \\
\hline \multirow[t]{4}{*}{ CC. } & HXHX & 4.7264 & & HXHXXX & 0.2248 \\
\hline & XXHX & 4.4845 & & XXHXHH & 0.2691 \\
\hline & XXHH & 4.3768 & & НХHХHН & 0.2691 \\
\hline & HXHH & 4.7778 & & НННХНH & 0.1738 \\
\hline \multirow[t]{2}{*}{$\mathrm{CX}$} & $\mathrm{HX}$ & 2.7476 & & HXHHHX & 0.1319 \\
\hline & $\mathrm{XX}$ & 2.7433 & & HXHHXX & 0.1319 \\
\hline $\mathbf{H C H}$ & $\mathbf{H H}$ & 0.5084 & & XXHHXX & 0.1319 \\
\hline HMH & ME & 0.5397 & & HHXXHH & 0.5867 \\
\hline \multirow[t]{3}{*}{$\mathrm{CMH}$} & HXME & 0.6105 & & HHXXHX & 0.5867 \\
\hline & HHME & 0.6172 & & HXXXHX & 0.5867 \\
\hline & XXME & 0.6433 & $\mathrm{MC}, \mathrm{CX}$ & MEHX & 0.6401 \\
\hline \multirow[t]{2}{*}{$\mathrm{MCH}$} & MEHX & 0.6719 & & MEXX & 0.4524 \\
\hline & MEHH & 0.7041 & $\mathrm{CC}, \mathrm{CX}$ & HXHX & 0.3959 \\
\hline \multirow[t]{5}{*}{$\mathrm{CCH}$} & HXHX & 0.6243 & & HHHX & 0.6401 \\
\hline & XXHX & 0.6063 & & XXHX & 0.3907 \\
\hline & XXHH & 0.6382 & & HXXX & 0.5832 \\
\hline & HXHH & 0.6467 & & HHXX & 0.6629 \\
\hline & HHHX & 0.6301 & $\mathrm{CX}, \mathrm{CX}$ & $\mathrm{XX}$ & 0.3644 \\
\hline \multirow[t]{2}{*}{ MCX } & MEHX & 1.1584 & CM,CMH & MEHX & 0.1845 \\
\hline & MEXX & 1.2696 & & MEHH & 0.1845 \\
\hline \multirow[t]{5}{*}{$\mathrm{CCX}$} & HXHX & 1.2246 & & MEXX & 0.1510 \\
\hline & XXHX & 1.5814 & $\mathrm{MC}, \mathrm{MCH}$ & MEHX & 0.2067 \\
\hline & HHHX & 0.8863 & & МEHH & 0.3065 \\
\hline & HXXX & 1.4457 & $\mathrm{MC}, \mathrm{MCX}$ & MEHX & 0.1003 \\
\hline & HHXX & 1.2605 & & MEXX & 0.0998 \\
\hline $\mathrm{HCX}$ & HX & 0.8751 & $\mathrm{MC}, \mathrm{MCC}$ & MEHXHX & 0.3584 \\
\hline $\mathrm{XCX}$ & $\mathrm{XX}$ & 1.0200 & & MEHXXX & 0.3584 \\
\hline \multirow[t]{7}{*}{$\mathrm{MCC}$} & MEHXHX & 1.0835 & & MEHXHH & 0.3334 \\
\hline & MEHXXX & 1.0047 & & MEXXHX & 0.3706 \\
\hline & MEHXHH & 1.0641 & & MEXXHH & 0.1293 \\
\hline & MEXXHX & 0.8865 & & MEHHHX & 0.3165 \\
\hline & MEXXHH & 0.8965 & & MEHHXX & 0.3165 \\
\hline & MEHHXX & 0.9987 & $\mathrm{CC}, \mathrm{CCC}$ & HXHXHX & 0.4994 \\
\hline & MEHHHX & 0.9987 & & HXHXXX & 0.4994 \\
\hline \multirow[t]{11}{*}{$\mathrm{CCC}$} & HXHXHX & 1.1746 & & XXHXHX & 0.4994 \\
\hline & HXHXXX & 1.1746 & & НХНХНH & 0.3744 \\
\hline & НХНХНН & 1.0918 & & HHHXXX & 0.3584 \\
\hline & XXHXHH & 1.0047 & & HHHXHX & 0.3584 \\
\hline & НННХНН & 0.9218 & & ХХНХНН & 0.1226 \\
\hline & HXHНHX & 1.2269 & & HXHHHX & 0.1700 \\
\hline & HXHHXX & 1.2269 & & HXHHXX & 0.1700 \\
\hline & XXHHXX & 1.2269 & & ХХНHНХ & 0.1700 \\
\hline & HHXXHH & 0.8965 & & XXHHXX & 0.1700 \\
\hline & HHXXHX & 0.8865 & & HHXXHH & 0.2064 \\
\hline & HXXXHX & 0.8865 & & HHXXHX & 0.3706 \\
\hline \multirow[t]{3}{*}{ M.C } & MEHX & 0.1410 & & НХXXНH & 0.2912 \\
\hline & MEXX & 0.1410 & & $\mathrm{HXXXHX}$ & 0.2912 \\
\hline & MEHH & 0.0784 & & НHНXHH & 0.1200 \\
\hline \multirow[t]{4}{*}{ C.C } & HXHX & 0.2733 & $\mathrm{CC}, \mathrm{CCH}$ & HXHX & 0.0395 \\
\hline & HXXX & 0.2733 & & $\mathrm{XXHX}$ & 0.1000 \\
\hline & HXHH & 0.1432 & & HНHX & 0.1000 \\
\hline & ХХHН & 0.2733 & & HXHH & 0.1200 \\
\hline $\mathbf{M H}, \mathbf{M H}$ & ME & 0.0010 & & $\mathrm{XXHH}$ & 0.0483 \\
\hline $\mathrm{CH}, \mathrm{CH}$ & HH & 0.0167 & $(\mathrm{CC}, \mathrm{CCH}) \neq$ & $-\mathrm{HX}-$ & 0.1603 \\
\hline \multirow[t]{2}{*}{$\mathrm{MC}, \mathrm{CC}$} & MEHXHX & 0.2691 & $\mathrm{CC}, \mathrm{CCX}$ & HXHX & 0.2614 \\
\hline & MEHXXX & 0.2691 & & $\mathrm{XXHX}$ & 0.2022 \\
\hline
\end{tabular}


Table 2 (continued)

\begin{tabular}{|c|c|c|c|c|c|}
\hline Constant* & Environ. $\dagger$ & Value & Constant* & Environ. $†$ & Value \\
\hline & HXXX & 0.1533 & & XXHXHX & -0.0160 \\
\hline & HHXX & 0.2061 & & HHHXHX & -0.0160 \\
\hline & HHHX & 0.2061 & & ННHХНH & -0.0160 \\
\hline \multirow[t]{2}{*}{$(\mathrm{CC}, \mathrm{CCX}) \ddagger$} & $-\mathrm{HX}-$ & 0.4070 & & HXXXHX & -0.0503 \\
\hline & $-\mathrm{XX}-$ & 0.4070 & & HXXXHH & -0.0503 \\
\hline \multirow[t]{7}{*}{$\mathrm{CC} . \mathrm{CCM}$} & HXHXME & 0.3744 & & HHXXHX & -0.0456 \\
\hline & HHHXME & 0.3754 & & HHXXHH & -0.0503 \\
\hline & XXHXME & 0.1226 & $\mathrm{CX}, \mathrm{CCX}^{\prime}$ & $\mathrm{HXXX}$ & -0.0645 \\
\hline & HXXXME & 0.2912 & & HHXX & -0.0664 \\
\hline & HHXXME & 0.2064 & $\mathrm{CX}, \mathrm{MCX}^{\prime}$ & MEXX & -0.0893 \\
\hline & HXHHME & 0.2064 & $\mathrm{CX}, \mathrm{CCC}$ & HXHXHX & -0.0549 \\
\hline & XXHHME & 0.2064 & & HXHXXX & -0.0549 \\
\hline $\mathrm{CX}, \mathrm{HCX}$ & $\mathrm{HX}$ & 0.0996 & & HXHXHH & -0.0878 \\
\hline $\mathrm{CX}, \mathrm{XCX}$ & $\mathrm{XX}$ & 0.2099 & & НHНXНH & -0.1500 \\
\hline \multirow[t]{2}{*}{$\mathrm{CX}, \mathrm{MCX}$} & MEHX & 0.3922 & & HHXXHH & -0.0480 \\
\hline & MEXX & 0.4052 & & HHXXHX & -0.1793 \\
\hline \multirow[t]{5}{*}{$\mathrm{CX}, \mathrm{CCX}$} & HXHX & 0.5727 & & HXXXHX & -0.1793 \\
\hline & XXHX & 0.6100 & НMH,НMH & $\mathrm{ME}$ & 0.0091 \\
\hline & $\mathrm{HXXX}$ & 0.6635 & $\mathrm{HMH}, \mathrm{CMH}$ & MEHX & 0.0039 \\
\hline & HHHX & 0.4095 & & MEXX & 0.0182 \\
\hline & HHXX & 0.5611 & & MEHH & 0.0039 \\
\hline \multirow[t]{5}{*}{$\mathrm{CX}, \mathrm{MCC}$} & MEHXHX & -0.0693 & $\mathrm{CMH}, \mathrm{CMH}$ & MEHX & -0.0237 \\
\hline & MEHXXX & -0.1602 & & МЕHН & -0.0237 \\
\hline & MEHXHН & -0.0300 & & MEXX & -0.0212 \\
\hline & MEXXHX & -0.1793 & $\mathrm{CCH}, \mathrm{CCH}^{\prime}$ & НХHН & -0.0300 \\
\hline & MEXXHН & -0.0840 & & XXHH & -0.0423 \\
\hline \multirow[t]{5}{*}{$\mathrm{MC}, \mathrm{HCC}$} & MEHXHX & -0.0256 & & НННН & -0.0400 \\
\hline & MEHHHX & -0.0218 & $\mathbf{M C H}, \mathrm{MCH}^{\prime}$ & MEHH & -0.0032 \\
\hline & MEHHXX & -0.0256 & $\mathrm{MCH}, \mathrm{HCX}$ & MEHX & 0.1483 \\
\hline & MEHXXX & -0.0218 & $\mathrm{CCH}, \mathrm{HCX}$ & HXHX & 0.1583 \\
\hline & MEHXHH & -0.0730 & & XXHX & 0.1022 \\
\hline \multirow[t]{6}{*}{$\mathrm{CC}, \mathrm{HCM}$} & HXHXME & -0.0803 & & HHHX & 0.1528 \\
\hline & XXHXME & -0.0865 & $(\mathrm{CCH}, \mathrm{HCX}) \ddagger$ & $-\mathrm{HX}-$ & 0.1507 \\
\hline & HHHXME & -0.1443 & $\mathrm{MCH}, \mathrm{HCC}$ & MEHXHX & 0.0293 \\
\hline & HXHHME & -0.0543 & & MEHXXX & 0.0240 \\
\hline & ХХНHМЕ & -0.0400 & & MEHXHH & 0.0338 \\
\hline & HНHНME & -0.0543 & & MEHHHX & 0.0240 \\
\hline \multirow[t]{12}{*}{$\mathrm{CC}, \mathrm{HCC}^{\prime}$} & HXHXHX & -0.0142 & & MEHHXX & 0.0440 \\
\hline & HXHXXX & -0.0142 & $\mathrm{CCH}, \mathrm{HCC}^{\prime}$ & HXHXHX & 0.0135 \\
\hline & XXHXHX & -0.0142 & & HXHXHH & 0.0293 \\
\hline & НХНХНH & -0.0803 & & HXHXXX & 0.0135 \\
\hline & HHHXXX & -0.0218 & & НHНХНH & 0.0000 \\
\hline & НHНXHX & -0.0256 & & HHHXXX & 0.0240 \\
\hline & НХHНHX & -0.0862 & & HXHHHX & 0.0036 \\
\hline & HXHHXX & -0.0862 & & HXHHXX & 0.0036 \\
\hline & ХХНHНX & -0.0862 & & XXHHXX & 0.0036 \\
\hline & XXHHXX & -0.0862 & $\mathrm{MCH}, \mathrm{MCX}$ & MEHX & 0.0999 \\
\hline & НННХНН & -0.0698 & $\mathrm{CCH}, \mathrm{CCX}$ & HXHX & -0.2683 \\
\hline & XXHXHH & -0.0865 & & HHHX & -0.1945 \\
\hline \multirow[t]{5}{*}{$\mathrm{MC}, \mathrm{XCC}$} & MEHXHX & -0.0130 & & $\mathrm{XXHX}$ & -0.1000 \\
\hline & MEHXHH & -0.0130 & MCX,HCX & MEHX & -0.0551 \\
\hline & MEHXXX & 0.0000 & CCX,HCX & HXHX & -0.1603 \\
\hline & MEXXHX & -0.0456 & & HHHX & -0.0111 \\
\hline & MEXXHH & -0.0651 & & XXHX & -0.3567 \\
\hline \multirow[t]{5}{*}{$\mathrm{CC}, \mathrm{XCM}$} & HXHXME & -0.0240 & $(\mathrm{CCX}, \mathrm{HCX}) \ddagger$ & $-\mathrm{HX}-$ & -0.0115 \\
\hline & XXHXME & 0.0000 & $\mathrm{MCX}, \mathrm{XCC}$ & MEHXHX & 0.0100 \\
\hline & HHHXME & -0.0300 & & MEHXXX & 0.0100 \\
\hline & HXXXME & -0.0503 & & MEHXHH & 0.0100 \\
\hline & HHXXME & -0.0503 & & MEXXHX & 0.0948 \\
\hline \multirow{3}{*}{$\mathrm{CC}, \mathrm{XCC}^{\prime}$} & $\mathrm{HXHXHX}$ & -0.0160 & & MEXXHН & 0.1233 \\
\hline & HXHXXX & -0.0160 & $\mathrm{MCC}, \mathrm{MCH}$ & MEHXHX & -0.0470 \\
\hline & HXHXHH & -0.0240 & & МЕНХНH & -0.2188 \\
\hline
\end{tabular}


Table 2 (continued)

\begin{tabular}{|c|c|c|c|c|c|}
\hline Constant* & Environ. $†$ & Value & Constant* & Environ. $\dagger$ & Value \\
\hline & MEHXXX & -0.0783 & & MEHXHX & 0.0080 \\
\hline & МЕHНHX & -0.1301 & & MEHXXX & 0.0080 \\
\hline & MEHHXX & -0.1301 & & МЕHХHH & 0.0080 \\
\hline \multirow[t]{5}{*}{$\mathrm{MCC}, \mathrm{CCH}$} & MEHXHX & -0.1364 & & MEHHXX & 0.0080 \\
\hline & МЕНХНH & -0.0540 & & MEHHHX & 0.0080 \\
\hline & MEHXXX & -0.0599 & & МЕНННН & 0.0080 \\
\hline & MEHHHX & -0.0898 & C.C,C.C & HXHXHX & 0.0080 \\
\hline & MEHHXX & -0.0898 & & НХHХHН & 0.0080 \\
\hline \multirow[t]{12}{*}{$\mathrm{CCC}, \mathrm{CCH}$} & НХНXHX & -0.1500 & & HXHXXX & 0.0080 \\
\hline & HXHXXX & -0.1500 & & HHHXHH & 0.0080 \\
\hline & НХНХНH & -0.1364 & & HНHXXX & 0.0080 \\
\hline & XXHXHX & -0.1500 & & HНXXHH & 0.0080 \\
\hline & ХХНХHН & -0.0783 & & HHXXHX & 0.0080 \\
\hline & HHHXXX & -0.0599 & & HXXXHX & 0.0080 \\
\hline & HHHXHX & -0.0470 & & НХНННХ & 0.0080 \\
\hline & HHHXHH & -0.1100 & & HXHHXX & 0.0080 \\
\hline & HXHHHX & -0.0688 & & XXHHXX & 0.0080 \\
\hline & HXHHXX & -0.0688 & $(\mathrm{HMC}, \mathrm{MCH})_{\mathrm{T}}$ & MEHX & 0.0706 \\
\hline & ХХННнХ & -0.0688 & & МЕHН & 0.1037 \\
\hline & XXHHXX & -0.0688 & $(\mathrm{HMC}, \mathrm{MCH})_{\mathrm{G}}$ & MEHX & -0.0194 \\
\hline \multirow[t]{5}{*}{ MCC,MCX } & MEHXHX & -0.0133 & & MEHH & -0.0460 \\
\hline & MEHXXX & -0.0236 & $(\mathrm{HMC}, \mathrm{MCXS})_{\mathrm{T}}$ & MEHX & -0.1542 \\
\hline & MEHXHH & -0.0107 & & MEXX & -0.2234 \\
\hline & MEXXHX & -0.0533 & $(\mathrm{HMC}, \mathrm{MCX})_{\mathrm{G}}$ & MEHX & -0.1222 \\
\hline & MEXXHH & -0.1442 & & MEXX & -0.0617 \\
\hline \multirow[t]{12}{*}{$\mathrm{CCC}, \mathrm{CCX}$} & HXHXHX & -0.4488 & $(\mathrm{CCM}, \mathrm{CMH})_{\mathrm{T}}$ & HXHXME & 0.0456 \\
\hline & HXHXXX & -0.4488 & & HНHXME & 0.1316 \\
\hline & HXHXHН & -0.3724 & & XXHXME & 0.0050 \\
\hline & HHHXXX & -0.1985 & & HXXXME & 0.0745 \\
\hline & НННХНН & -0.1473 & & HHXXME & 0.0580 \\
\hline & ХХНХНХ & -0.4488 & & HXHHME & 0.0545 \\
\hline & HНHXHX & -0.3724 & & XXHHME & 0.0545 \\
\hline & ХХНХНН & -0.3649 & $(\mathrm{CCM}, \mathrm{CMH})_{\mathrm{G}}$ & HXHXME & -0.0517 \\
\hline & HXXXHH & -0.1985 & & НHНXME & -0.0570 \\
\hline & HHXXHX & -0.0236 & & XXHXME & -0.0473 \\
\hline & HXXXHX & -0.4488 & & HXXXMF. & -0.0864 \\
\hline & ННХХНH & -0.0179 & & HHXXME & -0.0864 \\
\hline \multirow[t]{5}{*}{$\mathrm{MCC}, \mathrm{CCX}$} & MEHXHX & -0.3724 & & HXHHME & -0.0864 \\
\hline & MEHXHH & -0.3582 & & ХХHНME & -0.0864 \\
\hline & MEHXXX & -0.2174 & $(\mathrm{MCC}, \mathrm{CCX})_{\mathrm{T}}$ & MEHXHX & -0.1218 \\
\hline & MEXXHX & -0.1985 & & MEHXXX & -0.1218 \\
\hline & MEXXHH & -0.0179 & & MEHHHX & -0.1218 \\
\hline $\mathrm{MCX}, \mathrm{XCX}$ & MEXX & -0.1824 & & MEHHXX & -0.1826 \\
\hline \multirow[t]{2}{*}{$\mathrm{CCX}, \mathrm{XCX}$} & HXXX & -0.2858 & & MEXXHX & -0.0070 \\
\hline & HHXX & -0.1000 & & MEXXXX & -0.0070 \\
\hline \multirow[t]{2}{*}{$\mathrm{CCX}, \mathrm{CCX}^{\prime}$} & HXXX & 0.0492 & $(\mathrm{MCC}, \mathrm{CCX})_{\mathrm{G}}$ & MEHXHX & -0.0044 \\
\hline & HHXX & 0.2500 & & MEHXXX & -0.1435 \\
\hline \multirow[t]{2}{*}{$(\mathrm{MCX}, \mathrm{XCC}) \S$} & MEXXHX & 0.0533 & & МЕННHХ & -0.0299 \\
\hline & MEXXHH & 0.0040 & & MEHНXX & -0.0841 \\
\hline $\mathrm{MCX}, \mathrm{MCX}^{\prime}$ & MEXX & 0.1646 & & MEXXHX & -0.2498 \\
\hline \multirow[t]{7}{*}{$\mathrm{CCX} . \mathrm{XCC}$} & НХНХНХ & 0.0100 & & MEXXXX & -0.2498 \\
\hline & НХНХHН & 0.0100 & $(\mathrm{MCC}, \mathrm{CCH})_{\mathrm{T}}$ & MEHXHX & -0.1504 \\
\hline & HXHXXX & 0.0100 & & МЕНХНH & -0.1300 \\
\hline & НННХНН & 0.0100 & & MEXXHX & -0.2564 \\
\hline & HHXXHH & 0.0948 & & MEXXHН & -0.4449 \\
\hline & HHXXHX & 0.0948 & & MEHНHX & -0.1300 \\
\hline & HXXXHX & 0.0948 & $(\mathrm{MCC}, \mathrm{CCH})_{\mathrm{O}}$ & MEHXHX & -0.1168 \\
\hline \multirow[t]{3}{*}{$(\mathrm{CCX}, \mathrm{XCC}) \$$} & HXXXHX & 0.0533 & & MEHXHН & -0.0695 \\
\hline & HXXXHH & 0.0533 & & MEXXHX & -0.1815 \\
\hline & HHXXHH & 0.0040 & & MEXXHH & -0.1515 \\
\hline \multirow[t]{2}{*}{ M.C,C.C } & MEXXHX & 0.0080 & & MEHНHX & -0.1168 \\
\hline & MEXXHH & 0.0080 & $(\mathrm{HCC}, \mathrm{CCX})_{\mathrm{r}}$ & HXHX & -0.1261 \\
\hline
\end{tabular}


Table 2 (continued)

\begin{tabular}{|c|c|c|c|c|c|}
\hline Constant* & Environ.† & Value & Constant* & Environ. $f^{\dagger}$ & Value \\
\hline & HXXX & -0.1509 & & НHНХНХ & -0.0903 \\
\hline & HHHX & -0.1029 & & ХXHXHX & -0.0903 \\
\hline & HHXX & -0.2993 & & НХHXHН & -0.0903 \\
\hline \multirow[t]{4}{*}{$(\mathrm{HCC}, \mathrm{CCX})_{\mathrm{G}}$} & HXHX & -0.2988 & & НННХНН & -0.1300 \\
\hline & HXXX & -0.1723 & & XXHXHH & -0.0903 \\
\hline & HHHX & -0.1620 & & HXXXHX & -0.0903 \\
\hline & HHXX & -0.0208 & & HXXXHH & -0.0903 \\
\hline \multirow{2}{*}{$(\mathrm{XCC}, \mathrm{CCX})_{\mathrm{T}}$} & HXHX & -0.1579 & & HHXXHX & -0.0903 \\
\hline & HXXX & -0.2647 & & НHXXHН & -0.0903 \\
\hline \multirow[t]{2}{*}{$(\mathrm{XCC}, \mathrm{CCX})_{\mathrm{G}}$} & IIXHX & 0.0121 & & XXIIIIIX & -0.0930 \\
\hline & HXXX & 0.1028 & & HXHHHX & -0.0930 \\
\hline \multirow[t]{2}{*}{$(\mathrm{HCC}, \mathrm{CCH})_{\mathrm{T}}$} & HXHX & 0.0636 & $(\mathrm{CCC}, \mathrm{CCH})_{\mathrm{G}}$ & HXHXHX & -0.0966 \\
\hline & HХHH & 0.0852 & & HHНXНX & -0.0966 \\
\hline \multirow[t]{2}{*}{$(\mathrm{HCC}, \mathrm{CCH})_{\mathrm{G}}$} & HXHX & -0.0403 & & XXHXHX & -0.0966 \\
\hline & HXHH & -0.0403 & & HXHXHH & -0.0966 \\
\hline \multirow[t]{11}{*}{$(\mathrm{CCC}, \mathrm{CCX})_{\top}$} & IIXHXHX & -0.1218 & & HHIXXH & -0.0481 \\
\hline & HHHXHX & -0.1218 & & ХХHXHH & -0.1815 \\
\hline & XXHXHX & -0.1218 & & HXXXHX & -0.0966 \\
\hline & HXHXXX & -0.1218 & & IIXXXIIII & -0.1815 \\
\hline & HHHXXX & -0.1218 & & HHXXHX & -0.1815 \\
\hline & XXHHXX & -0.1668 & & HHXXHH & -0.1515 \\
\hline & IIXHIIXX & -0.1668 & & XXHHHX & -0.1246 \\
\hline & HXHHHX & -0.1668 & & HXHHHX & -0.1246 \\
\hline & ХХНННХ & -0.1668 & $(\mathrm{MCC}, \mathrm{CCC})_{\mathrm{T}}$ & $\mathrm{M}-\mathrm{C}-\mathrm{C}-\mathrm{C}-$ & -0.0889 \\
\hline & IIXXXIIX & -0.1218 & $(\mathrm{MCC}, \mathrm{CCC})_{\mathrm{G}}$ & $\mathrm{M}-\mathrm{C}-\mathrm{C}-\mathrm{C}-$ & -0.1302 \\
\hline & HHXXHX & -0.1218 & $(\mathrm{MCC}, \mathrm{CCM})_{\mathrm{T}}$ & МЕНHНHME & -0.0889 \\
\hline \multirow[t]{11}{*}{$(\mathrm{CCC}, \mathrm{CCX})_{\mathrm{G}}$} & HXHXHX & 0.0754 & & MEHXHHME & -0.0889 \\
\hline & НHНХНХ & 0.0754 & & MEIIXHXME & -0.0889 \\
\hline & XXHXHX & 0.0754 & & MEXXHHME & -0.0719 \\
\hline & HXHXXX & 0.0754 & & MEXXHXME & -0.0719 \\
\hline & IIHHXXX & 0.0754 & $(\mathrm{MCC}, \mathrm{CCM})_{\mathrm{G}}$ & MEHIIHHME & -0.1302 \\
\hline & XXHHXX & 0.0100 & & MEHXHHME & -0.1302 \\
\hline & HXHHXX & 0.0100 & & MEHXHXME & -0.1302 \\
\hline & IIXHHНX & 0.0100 & & MEXXIHHME & -0.3143 \\
\hline & ХХHНHX & 0.0100 & & MEXXHXME & -0.0932 \\
\hline & HXXXHX & 0.0754 & $(\mathrm{CCC}, \mathrm{CCC})_{\mathrm{T}}$ & $-\mathrm{C}-\mathrm{C}-\mathrm{C}-\mathrm{C}-$ & -0.0889 \\
\hline & III IXXI IX & 0.0754 & $(\mathrm{CCC}, \mathrm{CCC})_{\mathrm{G}}$ & $-C-C-C-C$ & -0.1302 \\
\hline$(\mathrm{CCC}, \mathrm{CCH})_{\mathrm{T}}$ & HXHXHX & -0.0903 & & & \\
\hline
\end{tabular}

${ }^{*} \mathrm{M}=$ Methyl carbon; $\mathrm{X}=$ chlorine; $\mathrm{T}=$ trans; $\mathrm{G}=$ gauche; $\mathrm{AB}=\mathrm{AB}$ stretch; $\mathrm{ABC}=\mathrm{ABC}$ bend; $\mathrm{Y}, \mathrm{Z}=\mathrm{YZ}$ interaction; $A . B=A B$ torsion. See Fig. 1 for designation of atoms in groups.

$\uparrow \mathrm{ME}=$ methyl group; $\mathrm{HH}=\mathrm{CH}_{2} ; \mathrm{HX}=\mathrm{CHCl} ; \mathrm{XX}=\mathrm{CCl}_{2}$.

$\ddagger(\mathrm{Y}, \mathrm{Z}) \ddagger=$ interaction for center $\mathrm{C}$ in a triplet containing $\mathrm{Cl}$ atoms (neighboring $\mathrm{C}$ atoms may have one or two $\mathrm{Cl}$ atoms).

$\S(\mathrm{Y}, \mathrm{Z}) \S=$ cross interaction; i.e. $(\mathrm{CCX}, \mathrm{XCC}) \S=\left(\mathrm{CCX}, \mathrm{X}^{\prime} \mathrm{CC}^{\prime}\right)$ in $\mathrm{C}-\mathrm{XCX}^{\prime}-\mathrm{C}^{\prime}-$.

Such an empirical refined force field is, of course, not unique. However, since the number of frequencies used in the refinement was about twice the number of independent force constants (which was about 260), we feel that the force field will give a good representation of the normal modes and their frequencies for a wide range of multiplychlorinated hydrocarbons.

\section{Carbon-Chlorine Stretch Freouencies}

Since normal mode calculations can be particularly useful in studying the conformation-sensitive carbon-chlorine stretch frequencies [3-5,9-11], we have given particular attention to the refinement of this part of the force field. In Table 3 we bring together the results for this part of the spectrum for all of the molecules used in our 
refinement (details for other parts of the spectrum can be found in the references cited or in [12]).

It can be seen from Table 3 that the agreement between observed and calculated frequencies is very good. For the 74 observed bands, the average error is $4.5 \mathrm{~cm}^{-1}$, with

Table 3. Observed and calculated $\mathrm{CCl}$ stretch frequencies of chlorinated hydrocarbons

\begin{tabular}{|c|c|c|c|c|}
\hline Mol.* & Obs." & Calc. & Conformer $\dagger$ & Potential energy distribution $¥$ \\
\hline \multirow[t]{3}{*}{ 2-CB } & 670 & 678 & $\mathrm{G}^{\prime}$ & $\mathrm{CX} \mathrm{s}(71) \mathrm{CHX} \operatorname{def}(19) \mathrm{CH}_{2} \operatorname{def}(13)$ \\
\hline & 627 & 630 & G & CX s(81) CX ib(19) CHX def(10) \\
\hline & 607 & 614 & $\mathrm{~T}$ & $\mathrm{CX} \mathrm{s}(92) \mathrm{CHX} \operatorname{def}(16)$ \\
\hline \multirow[t]{4}{*}{ 3-CP } & 668 & 684 & GG & $\mathrm{CX} \mathrm{s}(62) \mathrm{CH}_{2} \operatorname{def}(11) \mathrm{CX}$ ib(11) $\mathrm{CHX} \operatorname{def}(11)$ \\
\hline & 657 & 664 & $\mathrm{TG}^{\prime}$ & $\mathrm{CX} \mathrm{s}(63) \mathrm{CH}_{2} \mathrm{r}(15) \mathrm{CH}_{2} \operatorname{def}(14) \mathrm{CHX} \operatorname{def}(13)$ \\
\hline & 633 & 626 & TG & $\mathrm{CX} \mathrm{s}(72) \mathrm{CX}$ ib(19) $\mathrm{CH}_{2} \mathrm{r}(11) \mathrm{CH}_{2} \operatorname{def}(10)$ \\
\hline & 606 & 603 & TT & $\mathrm{CXs}(93) \mathrm{CHX} \operatorname{def}(11) \mathrm{CH}_{2} \mathrm{r}(10)$ \\
\hline \multirow[t]{7}{*}{ 2,4-DCP } & 718 & 718 & $G G(r)$ & $\mathrm{C}_{2} \times \mathrm{Ss}(30) \mathrm{C}_{4} \mathrm{Xs}(30) \mathrm{CH}_{2} \operatorname{def}(17)$ \\
\hline & 682 & 678 & $\mathrm{TG}(\mathbf{r})$ & $\mathrm{C}_{4} \mathrm{Xs}(73) \mathrm{C}_{4} \mathrm{HX} \operatorname{def}(18) \mathrm{CH}_{2} \operatorname{def}(10)$ \\
\hline & 631 & 624 & TT(r) & $\mathrm{C}_{2} \mathrm{Xs}(47) \mathrm{C}_{4} \mathrm{Xs}(47)$ \\
\hline & 611 & 615 & TT(r) & $\mathrm{C}_{2} \mathrm{Xs}(47) \mathrm{C}_{4} \mathrm{Xs}(47) \mathrm{C}_{2} \mathrm{HX} \operatorname{def}(11) \mathrm{C}_{4} \mathrm{HX} \operatorname{def}(11)$ \\
\hline & 685 & 679 & $\mathrm{TG}(\mathrm{m})$ & $\mathrm{C}_{4} \mathrm{Xs}(72) \mathrm{C}_{4} \mathrm{HX} \operatorname{def}(19) \mathrm{CH}_{2} \operatorname{def}(11)$ \\
\hline & 645 & 635 & $\mathrm{TG}^{\prime}(\mathrm{m})$ & $\mathrm{C}_{4} \mathrm{Xs}(67) \mathrm{C}_{4} \mathrm{Xib}(17) \mathrm{C}_{2} \mathrm{Xs}$ s(11) $\mathrm{C}_{4} \mathrm{HX} \operatorname{def}(10)$ \\
\hline & 618 & 619 & $\mathrm{TG}(\mathrm{m})$ & $\mathrm{C}_{2} \mathrm{Xs}(94) \mathrm{C}_{2} \mathrm{HX} \operatorname{def}(17)$ \\
\hline \multirow[t]{19}{*}{$2,4,6-\mathrm{TCH}$} & 725 & 724 & TTGG(s) & $\mathrm{C}_{6} \mathrm{Xs}(28) \mathrm{C}_{4} \mathrm{Xs}(26) \mathrm{C}_{5} \mathrm{H}_{2} \operatorname{def}(20) \mathrm{C}_{6} \mathrm{HX} \operatorname{def}(10)$ \\
\hline & 632 & 631 & TTGG(s) & $\mathrm{C}_{6} \mathrm{Xs}(44) \mathrm{C}_{4} \mathrm{Xs}(40) \mathrm{C}_{2} \mathrm{Xs}(14)$ \\
\hline & 632 & 628 & TTTT(s) & $\mathrm{C}_{2} \mathrm{Xs}(35) \mathrm{C}_{6} \mathrm{Xs}(35) \mathrm{C}_{4} \mathrm{Xs}(26)$ \\
\hline & 632 & 625 & TTTT(s) & $\mathrm{C}_{2} \mathrm{Xs}(39) \mathrm{C}_{6} \mathrm{Xs}(39) \mathrm{C}_{2} \mathrm{HX} \operatorname{def}(11) \mathrm{C}_{6} \mathrm{HX} \operatorname{def}(11)$ \\
\hline & 608 & 618 & TTGG(s) & $\mathrm{C}_{2} \mathrm{Xs}(79) \mathrm{C}_{2} \mathrm{HX} \operatorname{def}(16) \mathrm{C}_{4} \mathrm{Xs}(10)$ \\
\hline & 608 & 610 & TTTT(s) & $\mathrm{C}_{4} \mathrm{Xs}(69) \mathrm{C}_{2} \mathrm{Xs}(13) \mathrm{C}_{6} \mathrm{Xs}(13) \mathrm{C}_{4} \mathrm{HX} \operatorname{def}(12)$ \\
\hline & 688 & 702 & G'TTG(i) & $\begin{array}{l}\mathrm{C}_{2} \mathrm{Xs}(28) \mathrm{C}_{6} \mathrm{Xs}(28) \mathrm{C}_{3} \mathrm{H}_{2} \operatorname{def}(12) \mathrm{C}_{5} \mathrm{H}_{2} \operatorname{def}(12) \\
\mathrm{C}_{2} \mathrm{HX} \operatorname{def}(11) \mathrm{C}_{6} \mathrm{HX} \operatorname{def}(11)\end{array}$ \\
\hline & 688 & 694 & TGTG(i) & $\mathrm{C}_{4} \mathrm{Xs}(30) \mathrm{C}_{6} \mathrm{Xs}(28)$ \\
\hline & & 670 & TGTG(i) & $\mathrm{C}_{6} \mathrm{Xs}(43) \mathrm{C}_{4} \mathrm{Xs}(35) \mathrm{C}_{6} \mathrm{HX} \operatorname{def}(10)$ \\
\hline & & 668 & $\mathrm{G}^{\prime} \mathrm{TTG}(\mathrm{i})$ & $\mathrm{C}_{2} \mathrm{Xs}(41) \mathrm{C}_{6} \mathrm{Xs}(41)$ \\
\hline & 635 & 637 & TTTT(i) & $\mathrm{C}_{4} \times \mathrm{s}(40) \mathrm{C}_{2} \mathrm{Xs}(28) \mathrm{C}_{f} \mathrm{Xs}(28)$ \\
\hline & 619 & 619 & TGTG(i) & $\mathrm{C}_{2} \mathrm{Xs}(92) \mathrm{C}_{2} \mathrm{HX} \operatorname{def}(17)$ \\
\hline & 614 & 614 & G'TTG(i) & $\mathrm{C}_{4} \mathrm{Xs}(94) \mathrm{C}_{4} \mathrm{HX} \operatorname{def}(13)$ \\
\hline & 688 & 689 & TTTG(h) & $\mathrm{C}_{6} \mathrm{Xs}(62) \mathrm{C}_{6} \mathrm{HX} \operatorname{def}(19) \mathrm{C}_{5} \mathrm{H}_{2} \operatorname{def}(16)$ \\
\hline & 688 & 685 & TTG'T(h) & $\mathrm{C}_{4} \mathrm{Xs}(63) \mathrm{C}_{4} \mathrm{HX} \operatorname{def}(14) \mathrm{C}_{5} \mathrm{H}_{2} \operatorname{def}(13)$ \\
\hline & 628 & 624 & TTTG(h) & $\mathrm{C}_{2} \mathrm{Xs}(68) \mathrm{C}_{4} \mathrm{Xs}(24) \mathrm{C}_{2} \mathrm{HX} \operatorname{def}(12)$ \\
\hline & 620 & 623 & TTG'T(h) & $C_{6} \times s(48) C_{2} \times s(46)$ \\
\hline & 620 & 616 & TTG'T(h) & $\mathrm{C}_{2} \mathrm{Xs(46)} \mathrm{C}_{6} \mathrm{Xs}(44)$ \\
\hline & 610 & 611 & TTTG(h) & $\mathrm{C}_{4} \mathrm{Xs}(71) \mathrm{C}_{2} \mathrm{Xs}(22) \mathrm{C}_{4} \mathrm{HX} \operatorname{def}(11)$ \\
\hline \multirow[t]{2}{*}{ PVC } & 640 & 636 & (s) & $\mathrm{CX} \mathrm{s}(96) \mathrm{CX} \mathrm{ib}(20) \mathrm{CHX} \operatorname{def}(12)$ \\
\hline & 604 & 609 & (s) & $\mathrm{CX} s(96) \mathrm{CHX} \operatorname{def}(26)$ \\
\hline \multirow[t]{13}{*}{ 2,3-DCB } & 703 & 706 & MRSM(T) & $\mathrm{CX} \mathrm{s(45)} \mathrm{CX} \mathrm{ib(20)} \mathrm{CHX} \mathrm{def(24)} \mathrm{CH}$ ob(21) M r2(21) \\
\hline & 652 & 655 & MRSM(T) & $\mathrm{CXs}(97)$ \\
\hline & 344 & 352 & MRSM(T) & $\mathrm{CX} s(48) \mathrm{CHX} \operatorname{def}(27)$ \\
\hline & 706 & 700 & MRRM(T) & $\mathrm{CX} \mathrm{s(50)} \mathrm{CX} \mathrm{ib(46)} \mathrm{M} \mathrm{r2(18)} \mathrm{CHX} \mathrm{def(14)}$ \\
\hline & 597 & 602 & MRRM(T) & $\mathrm{CX} \mathrm{s}(94) \mathrm{CHX} \operatorname{def}(18)$ \\
\hline & 519 & 511 & MRRM(T) & $\mathrm{CX} \mathrm{s}(50) \mathrm{CX} \mathrm{ib}(26) \mathrm{CX}$ ob(12) \\
\hline & 697 & 708 & $\operatorname{MRSM}(G)$ & CX s(59) CHX def(34) CX ib(13) M r2(11) \\
\hline & 650 & 649 & $\operatorname{MRSM}(G)$ & $\mathrm{CX} \mathrm{s}(57) \mathrm{CX}$ ib(38) $\mathrm{CHX} \operatorname{def}(17)$ \\
\hline & 532 & 529 & MRSM(G) & $\mathrm{CX} \mathrm{s}(42) \mathrm{CX} \mathrm{ib}(36)$ \\
\hline & 420 & 427 & $\operatorname{MRSM}(G)$ & $\mathrm{CX} \mathrm{s}(33) \mathrm{CX}$ ob(17) $\mathrm{CX}$ ib(15) \\
\hline & 729 & 723 & $\operatorname{MRRM}(\mathbf{G})$ & $\mathrm{CX} \mathrm{s}(46) \mathrm{CHX} \operatorname{def}(38) \mathrm{CH}$ ob(18) $\mathrm{M}$ r2(18) $\mathrm{CX}$ ib(18) \\
\hline & 650 & 653 & MRRM(G) & $\mathrm{CX} \mathrm{s}(98)$ \\
\hline & 370 & 375 & $\operatorname{MRRM}(G)$ & $\mathrm{CX} \mathrm{s(42)} \mathrm{CHX} \mathrm{def(16)} \mathrm{CX} \mathrm{ib(16)}$ \\
\hline \multirow[t]{7}{*}{ 2,3,4-ТCP } & 765 & 765 & $\mathrm{TT}(\mathrm{r})$ & $\mathrm{C}_{3} \mathrm{X}$ ib(28) $\mathrm{C}_{3} \mathrm{X} \mathrm{s}(21) \mathrm{C}_{2} \mathrm{H} \mathrm{ob}(10) \mathrm{C}_{4} \mathrm{Xs}(10)$ \\
\hline & 673 & 672 & $\mathrm{TT}(\mathrm{r})$ & $C_{2} X s(69) C_{2} H X \operatorname{def}(15) C_{3} X s(10)$ \\
\hline & 626 & 616 & $\mathrm{TT}(\mathrm{r})$ & $\mathrm{C}_{4} \mathrm{Xs}(68) \mathrm{C}_{3} \mathrm{Xs}(13) \mathrm{C}_{4} \mathrm{HX} \operatorname{def}(11) \mathrm{C}_{4} \mathrm{Xib}(10)$ \\
\hline & 553 & 549 & TT(r) & $\mathrm{C}_{3} \mathrm{Xs}(17) \mathrm{C}_{4} \mathrm{Xs}(16) \mathrm{C}_{4} \mathrm{Xib}(13)$ \\
\hline & 745 & 742 & $\mathrm{TG}(\mathrm{m})$ & $\begin{array}{l}C_{3} X s(24) C_{3} H X \operatorname{def}(20) C_{3} X \text { ib }(14) C_{4} X s(13) \\
C_{2} X i b(10)\end{array}$ \\
\hline & 697 & 701 & $\mathrm{TG}(\mathrm{m})$ & $\mathrm{C}_{4} \mathrm{Xs}(42) \mathrm{C}_{4} \mathrm{HX} \operatorname{def}(20) \mathrm{C}_{3} \mathrm{X}$ ib(14) $\mathrm{C}_{2} \mathrm{Xs}(12)$ \\
\hline & 668 & 672 & $\mathrm{TG}(\mathrm{m})$ & $\mathrm{C}_{2} \mathrm{Xs}(57) \mathrm{C}_{3} \mathrm{Xs}(17) \mathrm{C}_{2} \mathrm{HX} \operatorname{def}(13)$ \\
\hline
\end{tabular}


Table 3 (continued)

\begin{tabular}{|c|c|c|c|c|}
\hline Mol.* & Obs. ${ }^{*}$ & Calc. & Confermer $\dagger$ & Potential energy distribution $\ddagger$ \\
\hline & 552 & 553 & $\mathrm{TG}(\mathrm{m})$ & $\mathrm{C}_{4} \mathrm{X} \mathrm{ib}(33) \mathrm{C}_{3} \mathrm{Xs}(26) \mathrm{C}_{3} \mathrm{Xob}(10)$ \\
\hline \multirow[t]{2}{*}{ 2,2-DCPr } & 656 & 653 & & $\mathrm{CX}_{2}$ as (97) $\mathrm{CX}_{2} \mathrm{r}(44) \mathrm{M}$ r2(13) \\
\hline & 559 & 561 & & $\mathrm{CX}_{2} \operatorname{ss}(66) \mathrm{CX}_{2} \operatorname{def}(11)$ \\
\hline \multirow[t]{6}{*}{ 2,2-DCB } & 645 & 646 & $\mathrm{~T}$ & $\mathrm{CX}_{2}$ as $(114) \mathrm{CX}_{2} \mathrm{r}(56)$ \\
\hline & 544 & 547 & $\mathrm{~T}$ & $\mathrm{CX}_{2} \mathrm{ss}(81) \mathrm{CX}_{2} \mathrm{~b}(13)$ \\
\hline & 276 & 269 & $\mathrm{~T}$ & $C X_{2} b(80) C X_{2} s s(16) C X_{2} \operatorname{def}(10)$ \\
\hline & 692 & 691 & G & $\mathrm{CX}_{2}$ as $(85) \mathrm{CX}_{2} \mathrm{r}(50) \mathrm{CH}_{2} \operatorname{def}(16)$ \\
\hline & 567 & 569 & $\mathbf{G}$ & $\mathrm{CX}_{2}$ ss(59) $\mathrm{CX}_{2}$ as(17) $\mathrm{CX}_{2} \operatorname{def}(14) \mathrm{CX}_{2} \mathrm{~b}(10)$ \\
\hline & 413 & 417 & G & $\mathrm{CX}_{2} \mathrm{w}(26) \mathrm{CX}_{2} \mathrm{ss}(18) \mathrm{CH}_{2} \operatorname{def}(16) \mathrm{CX}_{2}$ as $(10)$ \\
\hline \multirow[t]{12}{*}{$2,2,3-\mathrm{TCB}$} & 733 & 727 & $\mathrm{~T}$ & $\mathrm{CX}_{2} \mathrm{r}(52) \mathrm{CX}_{2}$ as (49) $\mathrm{CX} \mathrm{ib}(20) \mathrm{CX} \mathrm{s}(13)$ \\
\hline & 668 & 668 & $\mathrm{~T}$ & $\mathrm{CX} \mathrm{s}(60) \mathrm{CX}_{2}$ as $(20) \mathrm{CX}_{2} \mathrm{ss}(10)$ \\
\hline & 570 & 565 & $\mathrm{~T}$ & $\mathrm{CX}_{2} \mathrm{ss}(52) \mathrm{CX}_{2}$ as $(32) \mathrm{CX}_{2} \mathrm{~b}(11)$ \\
\hline & 361 & 362 & $\mathrm{~T}$ & $\mathrm{CXs}(21) \mathrm{CX}_{2}$ as(17) $\mathrm{CX}_{2} \mathrm{ss}(11)$ \\
\hline & 733 & 737 & G & $\mathrm{CX}_{2}$ as(53) $\mathrm{CX}_{2} \mathrm{r}(48) \mathrm{CHX} \operatorname{def}(26) \mathrm{CX} s(16)$ \\
\hline & 681 & 672 & $\mathrm{G}$ & $\mathrm{CX} \mathrm{s}(58) \mathrm{CX}_{2}$ as $(32)$ \\
\hline & 625 & 624 & $\mathrm{G}$ & $\mathrm{CX}_{2} \operatorname{ss}(44) \mathrm{CX}_{2} \operatorname{def}(26) \mathrm{CX}$ ib(26) \\
\hline & 439 & 437 & $\mathrm{G}$ & $\mathrm{CX}$ ib (23) $\mathrm{CX}_{2} \operatorname{ss}(21) \mathrm{CX}_{2} \operatorname{def}(10)$ \\
\hline & 721 & 723 & $\mathrm{G}^{\prime}$ & $\mathrm{CX}_{2}$ as (64) $\mathrm{CX}_{2} \mathrm{r}(48) \mathrm{CX}$ ib(17) \\
\hline & 699 & 687 & $\mathrm{G}^{\prime}$ & CXs(61) CHX def(23) $\mathrm{CX}_{2} \operatorname{def}(18)$ \\
\hline & 562 & 558 & $\mathrm{G}^{\prime}$ & $\mathrm{CX}_{2} \mathrm{ss}(60) \mathrm{CX}_{2}$ as $(23) \mathrm{CX}_{2} \mathrm{~b}(14)$ \\
\hline & 483 & 471 & $\mathrm{G}^{\prime}$ & $\mathrm{CX}_{2} \mathrm{w}(22) \mathrm{CX} \mathrm{s}(19) \mathrm{CX} \mathrm{ib}(16) \mathrm{CX}_{2}$ as(11) \\
\hline
\end{tabular}

*2-CB: 2-chlorobutane [4]; 3-CP: 3-chloropentane [4]; 2,4-DCP: 2,4-dichloropentane [5]; 2,4,6-TCH: 2,4,6-trichloroheptane [5]; PVC: poly(vinyl chloride) [9]; 2,3-DCB: 2,3-dichlorobutane [6]; 2,3,4-TCP: 2,3,4trichloropentane [1]; 2,2-DCPr: 2,2,-dichloropropane [7]; 2,2-DCB: 2,2-dichlorobutane [8]; 2,2,3-TCB: 2,2,3trichlorobutane [2].

$+\mathrm{T}=$ trans; $\mathrm{G}=$ gauche; $\mathrm{s}=$ syndiotactic; $\mathrm{i}=$ isotactic; $\mathrm{h}=$ heterotactic; $\mathrm{M}=$ methyl; for $\mathrm{R}$ and $\mathrm{S}$ see $[6]$; $\mathrm{r}=$ racemic $; \mathrm{m}=$ meso.

$\ddagger$ See Table 1 for definitions of local symmetry coordinates. $n$ in $\mathrm{C}_{n} \mathrm{X}$ refers to carbon atom number in molecular backbone. Contributions to PED of 10 or larger.

only 8 bands having errors of $10 \mathrm{~cm}^{-1}$ or more. (The error could probably be reduced if non-standard torsion angles were used for some of the conformers [4].) This force field should therefore be suitable for predicting the normal mode frequencies of different conformations of multiply-chlorinated hydrocarbons, including polymers.

Acknowledgements - This research was supported by the Polymers Program of NSF, grant DMR-8806975, and by a Macromolecular Research Center fellowship to one of us (S.H.C.).

\section{REFERENCES}

[1] S. H. Chough and S. Krimm, Spectrochim. Acta 46A, 1405 (1990).

[2] S. H. Chough and S. Krimm, Spectrochim. Acta 46A, 1419 (1990).

[3] C. G. Opaskar and S. Krimm, Spectrochim. Acta 23A, 2261 (1967).

[4] W. H. Moore and S. Krimm, Spectrochim. Acta 29A, 2025 (1973).

[5] W. H. Moore and S. Krimm, J. Molec. Spectrosc. 51, 1 (1974).

[6] X. Jing and S. Krimm, Spectrochim. Acta 39A, 251 (1983).

[7] P. G. Berlandier, J. L. Bribes and R. G. V. Tabacik, J. Chim. Phys. 75, 202 (1978).

[8] K. Ohno, Y. Shiro and H. Murata, Bull. Chem. Soc. Jap. 47, 2692 (1974).

[9] W. H. Moore and S. Krimm, Die Makromol. Chem. Suppl. 1, 491 (1975).

[10] S. Mizushima, T. Shimanouchi, K. Nakamura, M. Hayashi and S. Tsuchiya, J. Chem. Phys. 26, 970 (1957).

[11] J. J. Shipman, V. L. Folt and S. Krimm, Spectrochim. Acta 18, 1603 (1962).

[12] S. H. Chough, PhD thesis, University of Michigan (1988). 